Check for updates

Cite this: RSC Adv., 2019, 9, 1222

Received 19th October 2018 Accepted 19th December 2018

DOI: $10.1039 / c 8 r a 08662 a$

rsc.li/rsc-advances

\section{Morroniside alleviates coxsackievirus B3-induced myocardial damage apoptosis via restraining NLRP3 inflammasome activation}

\author{
Weidong Li, ${ }^{\text {ab }}$ Mao Chen, (D) ${ }^{* a}$ Lishuai Xu, ${ }^{c}$ Zhan Lv, ${ }^{b}$ Li Chen, ${ }^{b}$ Yiling Li ${ }^{d}$ \\ and WenFen $\mathrm{He}^{\mathrm{b}}$
}

Coxsackievirus B3 (CVB3)-induced myocardial damage always leads to serious heart failure by inducing cardiac injury. NLRP3 inflammasome activation has been identified as a central player in the pathogenesis of CVB3-induced viral myocarditis. Therefore, restraining NLRP3 inflammasome activation has been supposed to significantly alleviate the severity of myocardial damage and improve cardiac function. Morroniside (MR), one of the main iridoid glycosides, has the ability to depress the production of reactive oxygen species (ROS) and restrain the expression of caspase-3 and -9. Of importance, ROS and caspase are essential for NLRP3 inflammasome activation in response to CVB3 infection. Therefore, in the present study, MR was selected as a model drug to alleviate CVB3-induced myocardial damage. The results of cardiac function index determination showed that abnormal indexes including mean arterial pressure, heart rate, and left ventricular systolic pressure of myocardial damage rats could be recovered by treating with MR. Such results can be further verified by histopathological evaluation, with the heart tissues of CVB3-infected rats displaying the most amount of H\&E and TUNEL positive cells. The underlying mechanism by which MR improves the cardiac function was subsequently investigated. The detection of various gene levels indicated that NLRP3 inflammasome activation was inhibited by MR through down-regulating the expression of pro-inflammatory cytokines: interleukin (IL)- $\beta$ and IL-18, the pivotal factors that lead to inflammatory responses. More importantly, the related genes, cardiac function indexes, and various myocardial damage markers of normal rats treated with MR did not exhibit any obvious changes compared with the control group, indicating a satisfactory biocompatibility of MR. In summary, MR holds a great potential in the alleviation of CVB3-induced myocardial damage with a negligible cytotoxicity to normal heart tissues.

\section{Introduction}

Viral myocarditis, characterized with local and systemic inflammatory response, is an inflammatory disease and the major cause of sudden cardiac death in young adults. ${ }^{1,2}$ It is widely accepted that the predominant cause of viral myocarditis is coxsackievirus B3 (CVB3) which has been suggested as the most common pathogen for heart failure. ${ }^{3,4}$ More importantly, it has been reported previously that CVB3-induced acute myocarditis can progress to a dilated cardiomyopathy with a poor prognosis. ${ }^{5}$ Emerging evidence indicates that cardiac

${ }^{a}$ Department of Cardiology, Sichuan University, West China Hospital, No. 37 Guoxue Alley, Chengdu, 610041, Sichuan Province, China. E-mail: chenmaocc@outlook.com; Tel: +86-0817-2262404

${ }^{b}$ Department of Cardiology, Affiliated Hospital of North Sichuan Medical College, Sichuan Province, China

${ }^{c}$ Department of Ophthalmology, Affiliated Hospital of North Sichuan Medical College, Sichuan Province, China

${ }^{d}$ Department of Cardiology, Second Affiliated Hospital of North Sichuan Medical College, Sichuan Province, China damage is induced by CVB3 infection generally through the cardiomyocyte cytopathic effect and activating the reaction of the immune system..$^{6-8}$

Although the exactly pathogenesis of viral myocarditis is still elusive, the excessive innate immune response-induced inflammation has been identified as the major mechanism leading to cardiac injury. ${ }^{6}$ Nucleotide-binding oligomerization domain 2 (NOD2) belongs to the family of NOD-like receptors (NLR), and is closely associated with exacerbation of inflammation and cardiac remodeling.,90 In addition, NOD-like receptor pyrin domain-containing 3 (NLRP3) inflammasome is abundantly found on phagocytic antigen-presenting cells including macrophages and dendritic cells. ${ }^{11}$ Accumulating evidence has demonstrated that interaction of NLRP3 with apoptosis-associated speck-like protein containing CARD (ASC) can result in the maturation of interleukin (IL)- $\beta$ and IL-18 by inducing the auto-cleavage of caspase-1. ${ }^{\mathbf{1 2 - 1 4}}$ Importantly, IL-1 $\beta$ and IL-18 are members of the family of highly pro-inflammatory IL-1 cytokines which play a pivotal role in the exacerbation of inflammatory response. ${ }^{15,16}$ In this case, NLRP3 is supposed to 
be the most important inflammasome of CVB3-induced myocarditis and restraining NLRP3 inflammasome activation holds great potential for alleviating the severity of myocardial damage.

Morroniside (MR) belongs to an extensive group of iridoid glycosides, which are extracted from Cornus officinalis. ${ }^{\mathbf{1 7}, 18}$ Studies previously reported that the majority of iridoid glycosides possess many pharmacological activities such as mitigating vascular complications and cerebral ischemiareperfusion injury. ${ }^{19,20}$ Of importance, MR was thought to also have the ability to depress immunity by various mechanisms. ${ }^{21}$ Reports have demonstrated previously that co-incubation of MR with SHSY5Y cells can significantly restrict the formation of reactive oxygen species (ROS) which are crucial for inflammasome activation in response to $\mathrm{CVB} 3 .^{22,23}$ In addition, MR was also supposed to possess the capacity of depressing the activation of caspase-3 and -9 while upregulating Bcl-2. ${ }^{24,25}$ It is well known that inflammation is accompanied by apoptosis in various myocardial injuries. ${ }^{26-28}$ A previous study reported that the NLRP3 pathway triggered neuronal apoptosis in ischemic stroke mice. ${ }^{29}$ We suppose that MR can inhibit apoptosis via NLRP3 inflammasome inhibition.

Therefore, in the present study, we have explored the therapeutic efficacy of MR in CVB3-induced myocarditis using animal models. Besides, the underlying mechanisms of NLRP3 inflammasome inhibition and alleviation of myocardial damage were further evaluated.

\section{Materials and methods}

\section{Materials and rats}

MR (Fig. 1) was purchased from Selleck Inc (USA). Antibodies for cleaved Bax, Bcl-2, muscle hemoglobin (Mb), creatine kinase isoenzyme (CK-MB), cardiac troponin I (cTn I), NLRP3, ASC, caspase-3, IL-18, and IL- $\beta$ and HRP-conjugated secondary were purchased from Cell Signaling Technology Inc. (USA) or Abcam. Colorimetric TUNEL Apoptosis Assay Kit was obtained from Beyotime Institute of Biotechnology, Jiangsu, China.

Wild type Sprague-Dawley (SD) male rats (aged 6-8 weeks) were purchased from SAGE Laboratories (St. Louis, MO, USA). Rats were housed under constant conditions and free to water and aseptic food ad libitum during the experimental period.

\section{Establishment of the animal model}

The CVB3-induced myocardial animal models were established as reported previously. ${ }^{30}$ In brief, before injection, rats were anaesthetized by inhalation of $2.0 \mathrm{vol} \%$ isoflurane for $5 \mathrm{~min}$ using an automatic delivery system. SD rats were inoculated intraperitoneally with $100 \mu$ l RPMI 1640 medium containing 1 $\times 10^{5}$ plaque forming units of CVB3 virus once a day for a week which had been developed by a method similar to that of a former investigation. ${ }^{31}$

For drug administration, the rats were given MR or normal saline (NS) at the $2^{\text {nd }}$ day after inoculation. Thirty-six rats were randomly divided into four groups $(n=9)$ as follows: (1) control group: normal rats treated with NS; (2) MR group: normal rats

\section{Cornus officinalis Sieb. et Zucc.}

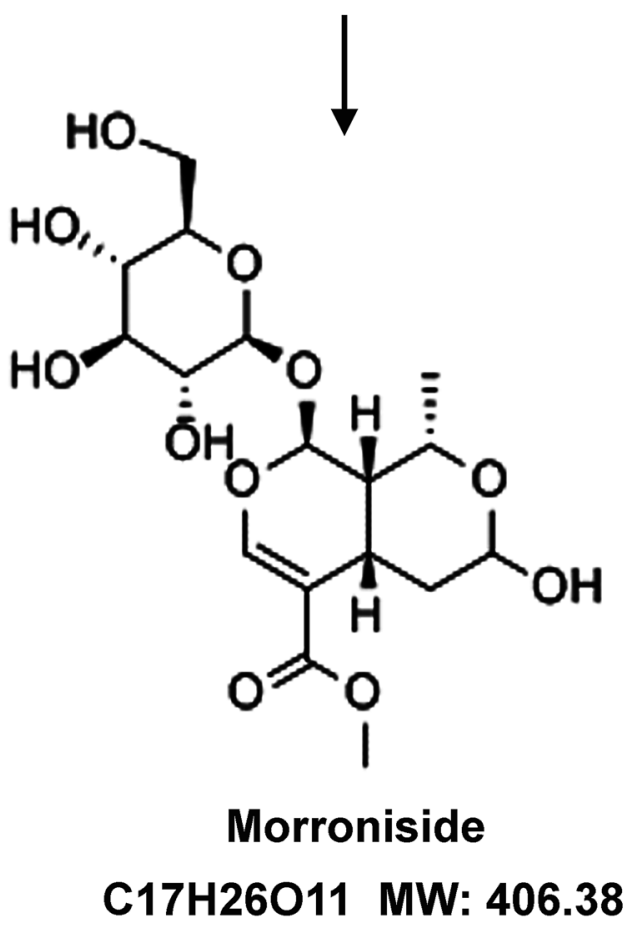

Fig. 1 Chemical structural, molecular formula, and molecular weight of morroniside.

treated with MR at a dosage of $20 \mathrm{mg} \mathrm{kg}^{-1}$; (3) CVB3 group: CVB3-induced myocardial rats received with or without agents; (4) CVB3 + MR group: CVB3-induced myocardial rats treated with MR (20 mg kg $\left.{ }^{-1}\right)$. Rats in the MR group and CVB3 + MR group were intraperitoneally injected with $20 \mathrm{mg} \mathrm{kg}^{-1} \mathrm{MR}$. Importantly, all the animal experiments were performed according to the NIH guidelines for the Care and Use of Laboratory Animals, and approved by the Animal Care and Use Committee of Affiliated Hospital of North Sichuan Medical College.

\section{Determination of cardiac function index}

After treatment with or without MR, the normal rats and CVB3induced rats were subjected to determination of cardiac function indexes mainly including mean arterial pressure (MAP), heart rate (HR), and left ventricular systolic pressure (LVSP).

\section{TUNEL analysis and H\&E staining}

Heart tissues of rats in each group were collected and immersed in acetone for fixing. Twenty-four hours later, $10 \mu \mathrm{m}$ slices were sectioned followed by apoptosis detection using Tdt-mediated dUTP nick-end labeling (TUNEL) according to the protocol of the manufacturer's instruction. The final results were obtained using an optical microscope (Olympus, Japan) by counting the total numbers of TUNEL positive nuclei. For hematoxylin and eosin (H\&E) staining analysis, the obtained hearts were embedded into paraffin before preparing $5 \mu \mathrm{m}$ tissue slices. 
Subsequently, the H\&E staining was performed using the standard protocol with a slight modification, ${ }^{32}$ and results were obtained using an optical microscope.

\section{Enzyme-linked immunosorbent assay (ELISA)}

To evaluate the expression of Mb, CK-MB, and cTn I and the levels of IL-18 and IL- $\beta$ in different heart tissues, all of the mice in each group were sacrificed. Finally, an ELISA kit (Elabscience, China) was applied to quantitatively evaluate the levels of various proteins.

\section{Reverse transcription polymerase chain reaction (RT-PCR)}

The total RNA from heart tissues of the CVB3-induced myocarditis model rats was extracted utilizing Trizol reagent (Takara, Japan) followed by reverse-transcribing into cDNAs via a Prime Script ${ }^{\mathrm{TM}}$ RT Master Mix kit (Takara, Japan). Subsequently, a Bio-Rad iQ iCycler detection system was applied to amplify the cDNA through the SYBR Green detection method. The expression of the specific NLRP3 transcripts was normalized to the expression of $\beta$-actin and measured using the $2^{-\Delta \Delta C_{t}}$ method. $\beta$-Actin, forward primer $5^{\prime}$-TCACCGAGCGCGGCT- $3^{\prime}$, and reverse primer $5^{\prime}$-TAATGTCACGCACGATTTCCC-3'; NLRP3, forward primer $5^{\prime}$-ACGGCAAGTTCGAAAAAGGC- $3^{\prime}$, and reverse primer 5'-AGACCTCGGCAGAAGCTAGA-3'.

\section{Western blot assay}

After receiving treatment with MR or not, all the mice were sacrificed and the hearts were collected. Subsequently, the total protein was extracted followed by determination of protein concentration using a Micro BCATM Protein Assay Kit (Thermo Fisher, MA, USA). Thereafter, the extracted proteins were transferred into PVDF membranes and subjected to blocking and washing. To evaluate the expression of Bax, Bcl-2, ASC and caspase-3, corresponding primary antibodies (Abcam, Cambridge, UK) were introduced and co-incubated with the protein- transferred membranes at $4{ }^{\circ} \mathrm{C}$. After overnight incubation, the Bax, Bcl-2, ASC and caspase- 3 antibodies were cleared away and all of the membranes were interacted with secondary antibodies (Abcam, Cambridge, UK) for $1 \mathrm{~h}$ at room temperature. Finally, the immunoreactive bands were detected through an Odyssey infrared imaging system (Li-Cor Bioscience, NE, USA).

\section{Statistical analysis}

Statistical differences between various groups were determined by one-way analysis of variance (ANOVA), followed by a Dunnett's post hoc analysis for multi-group comparison. Statistical significance was defined as $P<0.05$.

\section{Results}

\section{Determination of cardiac function index}

The main cardiac function indexes of different rats were determined post-treatment with or without MR. As shown in Fig. 2, the levels of MAP, HR, and LVSP of normal rats which had been treated with MR did not display significant changes compared with the control group, suggesting a relatively good biocompatibility of MR. However, for the CVB3-induced myocardial animal models, the levels of relevant indexes were remarkably reduced. Interestingly, the levels of MAP, HR, and LVSP could be recovered obviously by treating with MR. These results together indicate that $\mathrm{MR}$ is safe enough to be used as a drug model and could significantly alleviate CVB3-induced myocardial damage.

\section{H\&E staining and TUNEL analysis}

The H\&E staining and TUNEL analysis were applied to investigate the treatment efficacy of MR in the CVB3-induced myocardial rats. The results displayed in Fig. 3A show that the heart tissues of CVB3-infected rats exhibited the most serious myocardial damage since a large amount of TUNEL positive
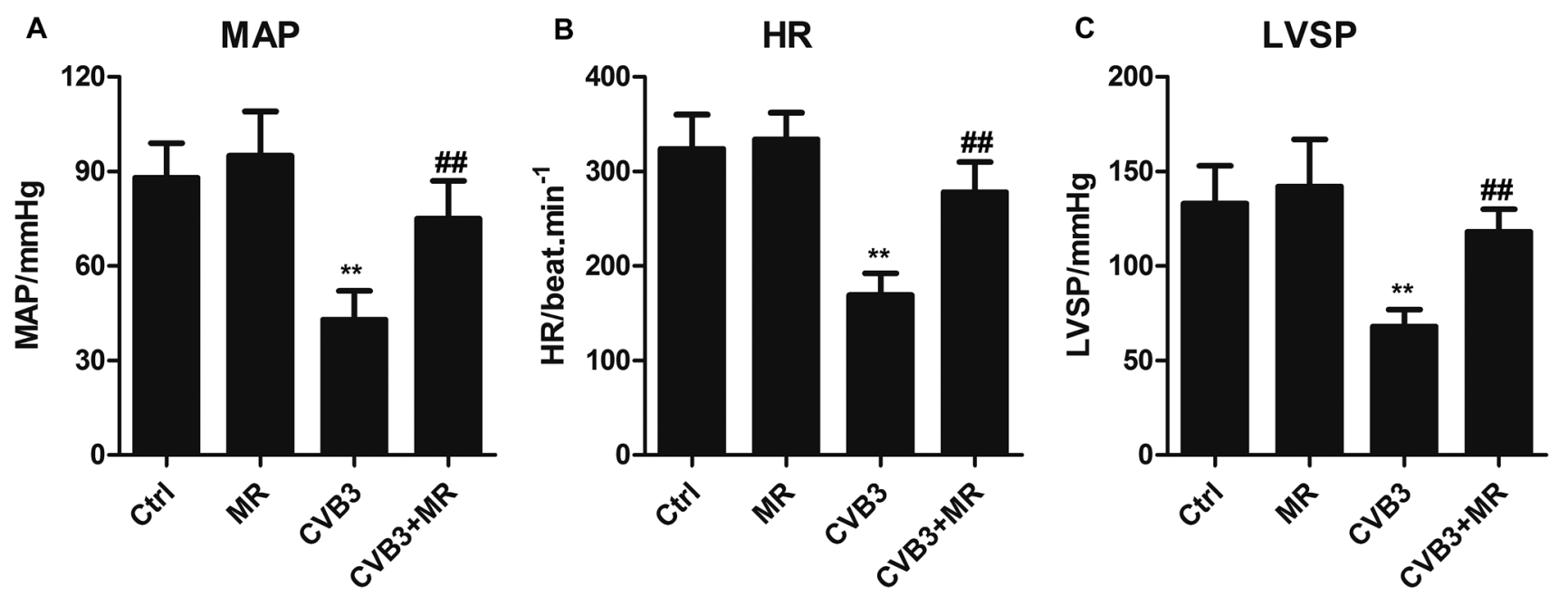

Fig. 2 Determination of cardiac function indexes. The cardiac function indexes of (A) MAP, (B) HR, and (C) LVSP of normal rats and CVB3induced myocardial rats post-treatment with or without MR $\left(20 \mathrm{mg} \mathrm{kg}^{-1}\right)$. **P $<0.01$ compared with control group, \#\# $P<0.01$ compared with control group. CVB3 = coxsackievirus B3, MR = morroniside, MAP = mean arterial pressure, HR $=$ heart rate, LVSP $=$ left ventricular systolic pressure. 
A

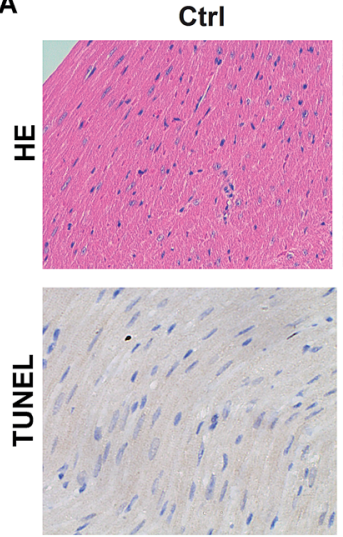

MR

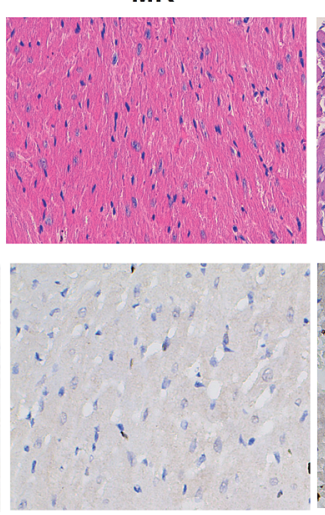

CVB3

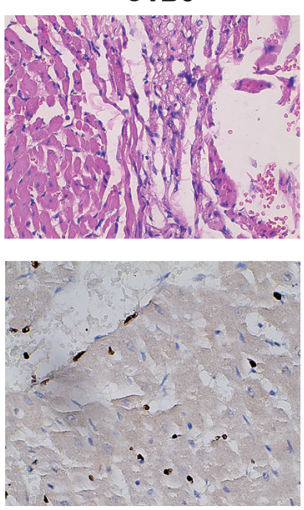

CVB3+MR

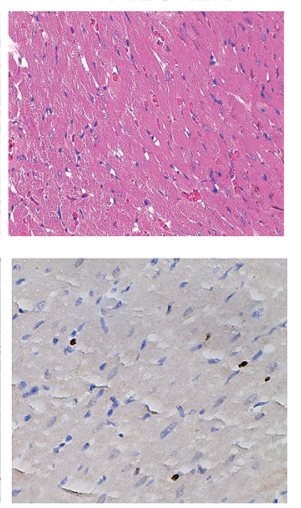

B

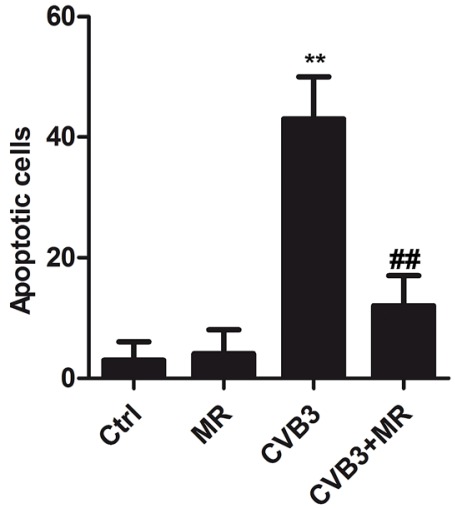

C

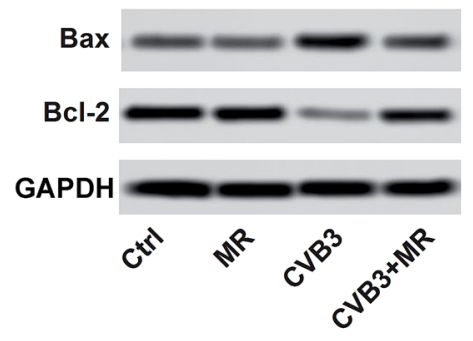

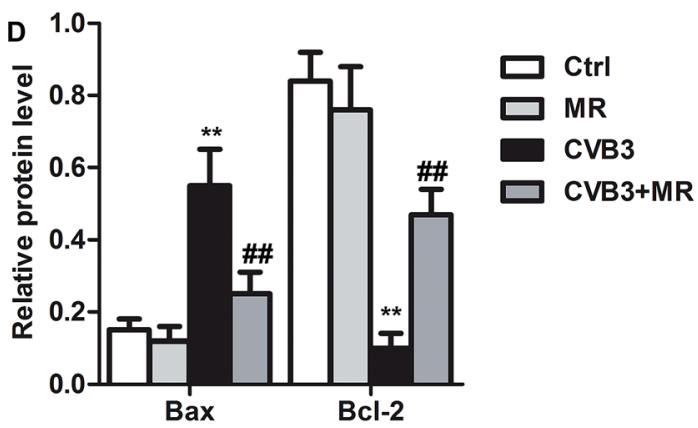

Fig. 3 Treatment efficacy of MR in CVB3-induced myocardial rats. All of the MR-treated rats received agents at a dosage of $20 \mathrm{mg} \mathrm{kg}^{-1}$. For comparison, normal rats and myocardial rats of other groups were treated with saline. (A) H\&E staining and TUNEL evaluation of heart tissues after preparing $5 \mu \mathrm{m}$ tissue slides. (B) Qualitative images obtained by the optical microscope (left) and the quantitative analysis of apoptotic cells performed by counting the total numbers of TUNEL positive cells (brown cells). Qualitative (C) and quantitative (D) evaluation of the Bax/Bcl-2 levels in each group. ${ }^{* * P}<0.01$ compared with control group, ${ }^{\# \#} P<0.01$ compared with control group. CVB3 $=$ coxsackievirus B3, MR $=$ morroniside, $\mathrm{HE}=$ hematoxylin and eosin.

nuclei could be seen under the optical microscope. Importantly, after treatment with MR, the total number of apoptotic cells were significantly decreased, indicating a strong myocardial damage inhibition ability of MR. In addition, to study the cytotoxicity of MR to normal myocardial cells, eighteen normal rats were randomly divided into two groups $(n=9)$ and administered with saline and MR $\left(20 \mathrm{mg} \mathrm{kg}^{-1}\right)$, respectively. Results shown in Fig. 3B demonstrate that MR did not show any obvious toxicity to normal myocardial cells compared with the control group, indicating a satisfactory safety of MR. Moreover, such results were further confirmed by the TUNEL analysis.

\section{Regulating the expression of Bax/Bcl-2 in myocardial rats by treating with MR}

As demonstrated previously, the expression of Bax/Bcl-2 in CVB3-induced myocardial rats could be significantly changed by MR treatment. ${ }^{23}$ For verification, a western blot assay was performed to determine the levels of Bax and Bcl-2 in heart tissues of rats treated by different strategies. As shown by the results in Fig. 3C, there is no significant difference of Bax/Bcl-2 expression between the control group and MR group (normal rats treated with MR). In contrast, the levels of Bax/Bcl-2 in the CVB3-induced rats were changed obviously after receiving MR therapy. Moreover, the levels of Bax, the depressor of Bcl-2, were remarkably up-regulated in the CVB3-induced rats. However, such expression can be reversed thoroughly by treating with MR with the activation of Bax being severely depressed and the levels of Bcl-2 be improved observably.

\section{Effect of MR on the levels of myocardial damage markers}

In order to investigate whether MR can alleviate the severity of myocardial damage and improve cardiac function, the major markers of myocardial damage including $\mathrm{Mb}$, CK-MB, and cTn I were determined. As demonstrated in Fig. 4, the levels of Mb, CK-MB, and cTn I are dramatically elevated after the rats were induced by CVB3. However, treatment with MR leads the CVB3induced rats displaying an obvious lower expression of myocardial damage markers compared with the untreated ones. More importantly, the myocardial damage markers of normal rats treated with MR did not show any significant difference compared with the control group, indicating that the cytotoxicity of MR to healthy heart tissues is negligible.

\section{Restraining NLRP3 inflammasome activation via treatment with MR}

Activation of NLRP3 inflammasome has been identified as the most predominant mechanism for CVB3 inducing myocardial damage. MR was selected as a model drug in the present study for its capacity to down-regulate the downstream genes of 
A

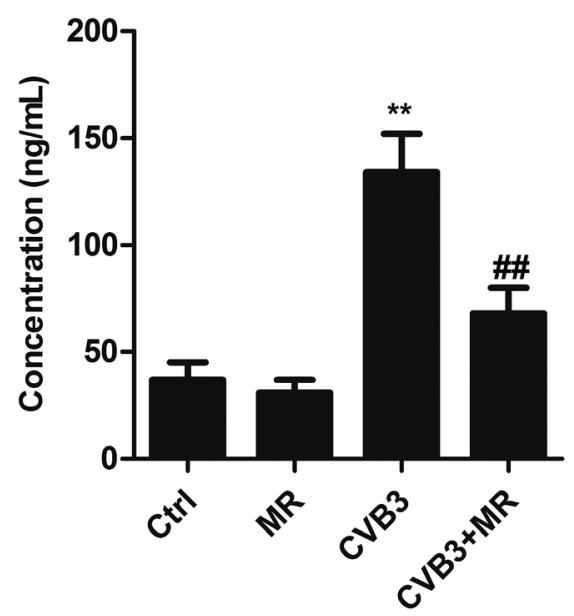

B

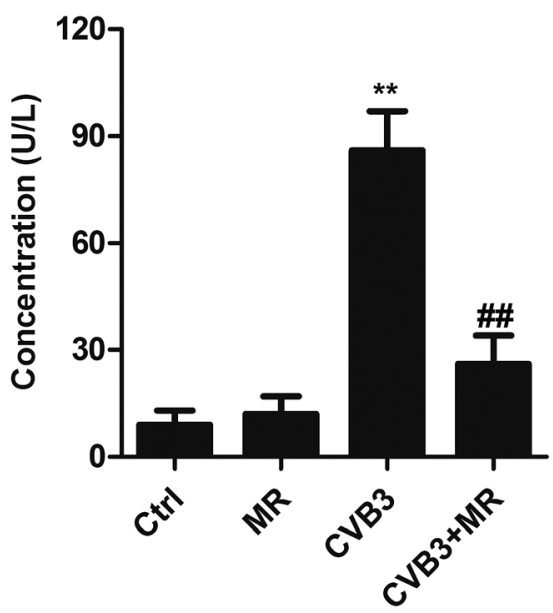

C

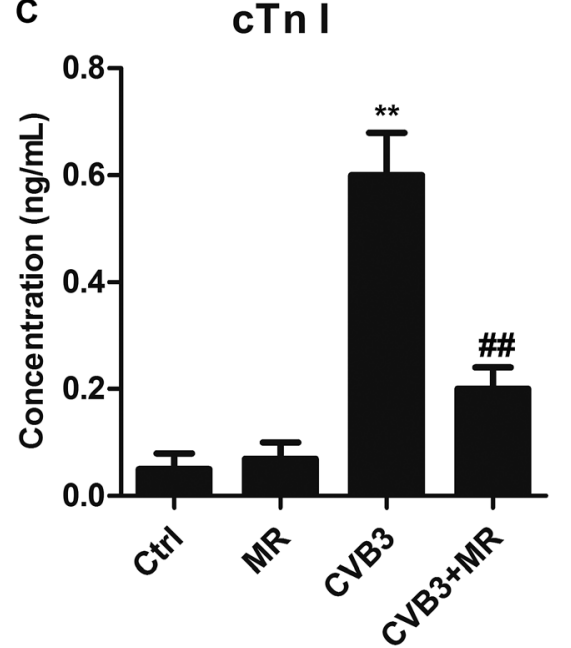

Fig. 4 Effect of MR on the levels of myocardial damage markers. The rat heart tissues of each group were harvested and subjected to determination of the levels of various myocardial damage markers: (A) Mb, (B) CK-MB, and (C) $\mathrm{CTn} I$. ** $P<0.01$ compared with control group, $\# \#<<$ 0.01 compared with control group. $C V B 3=$ coxsackievirus $B 3, M R=$ morroniside, $M b=$ muscle hemoglobin, $C K-M B=$ creatine kinase isoenzyme, cTn I = cardiac troponin I.

NLRP3 such as caspase-3 and -9. Based on such theory, we hypothesized that the activation of NLRP3 inflammasome can be significantly inhibited by treatment with MR. To verify this, the levels of NLRP3 in the heart tissues of each group were determined by RT-PCR experiments and western blot assay. As shown in Fig. 5A, results of RT-PCR indicated that the expression of NLRP3 in the CVB3-induced group was the highest, indicating a hyperactive NLRP3 inflammasome for the myocardial damage rats. Importantly, the levels of NLRP3, cleaved caspase- 1 and IL- $\beta$ were obviously down-regulated after treatment with MR, suggesting that the NLRP3 inflammasome activation can be restrained by treatment with MR. For further confirmation, a western blot assay was performed subsequently.
Results of qualitative and quantitative analysis shown in Fig. 5B and C were consistent with those of RT-PCR, with the CVB3 + MR group displaying lower levels of NLRP3 than the untreated group.

\section{Underlying mechanism of MR alleviating myocardial damage}

ASC and caspase- 3 are the downstream genes of NLRP3 and play an essential role in the maturation of IL- $\beta$ and IL-18 which are the main causes of inflammatory responses and myocardial damage. ${ }^{13,15}$ As demonstrated above, the activation of NLRP3 can be significantly depressed by MR treatment. In this case, the levels of ASC, caspase-3, IL- $\beta$, and IL-18 in the heart tissues of myocardial rats are supposed to be down-regulated after
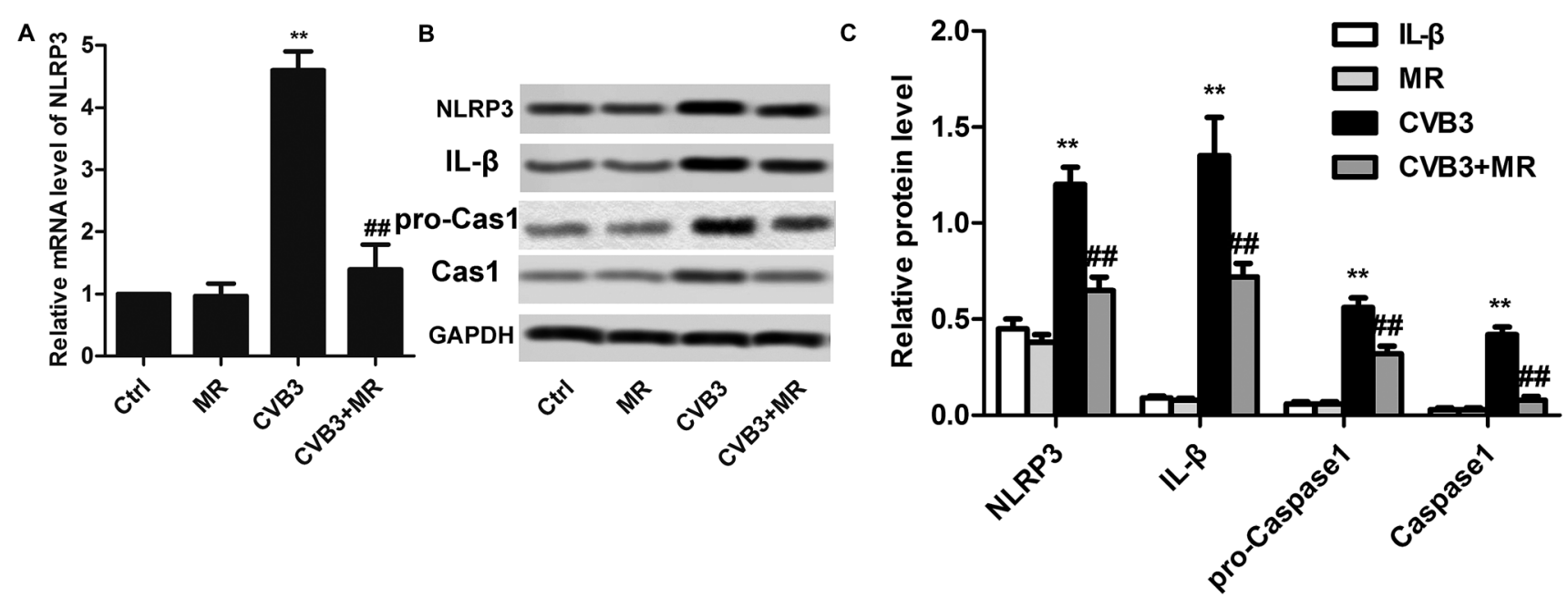

Fig. 5 Restraining NLRP3 inflammasome activation via treatment with MR. (A) Evaluation of NLRP3 levels in heart tissues of each group by performing RT-PCR experiments. (B) Qualitative analysis of treatment effect of MR on the expression of NLRP3, pro-caspase-1, cleaved caspase1 and IL- $\beta$ in different heart tissues. (C) Quantitative analysis of treatment effect of MR on the expression of NLRP3, pro-caspase-1, cleaved caspase- 1 and IL- $\beta$ in different heart tissues. ${ }^{*} P<0.01$ compared with control group, ${ }^{\# \# ~} P<0.01$ compared with control group. CVB3 $=$ coxsackievirus B3, MR = morroniside, NLRP3 $=$ NOD-like receptor pyrin domain-containing 3 . 


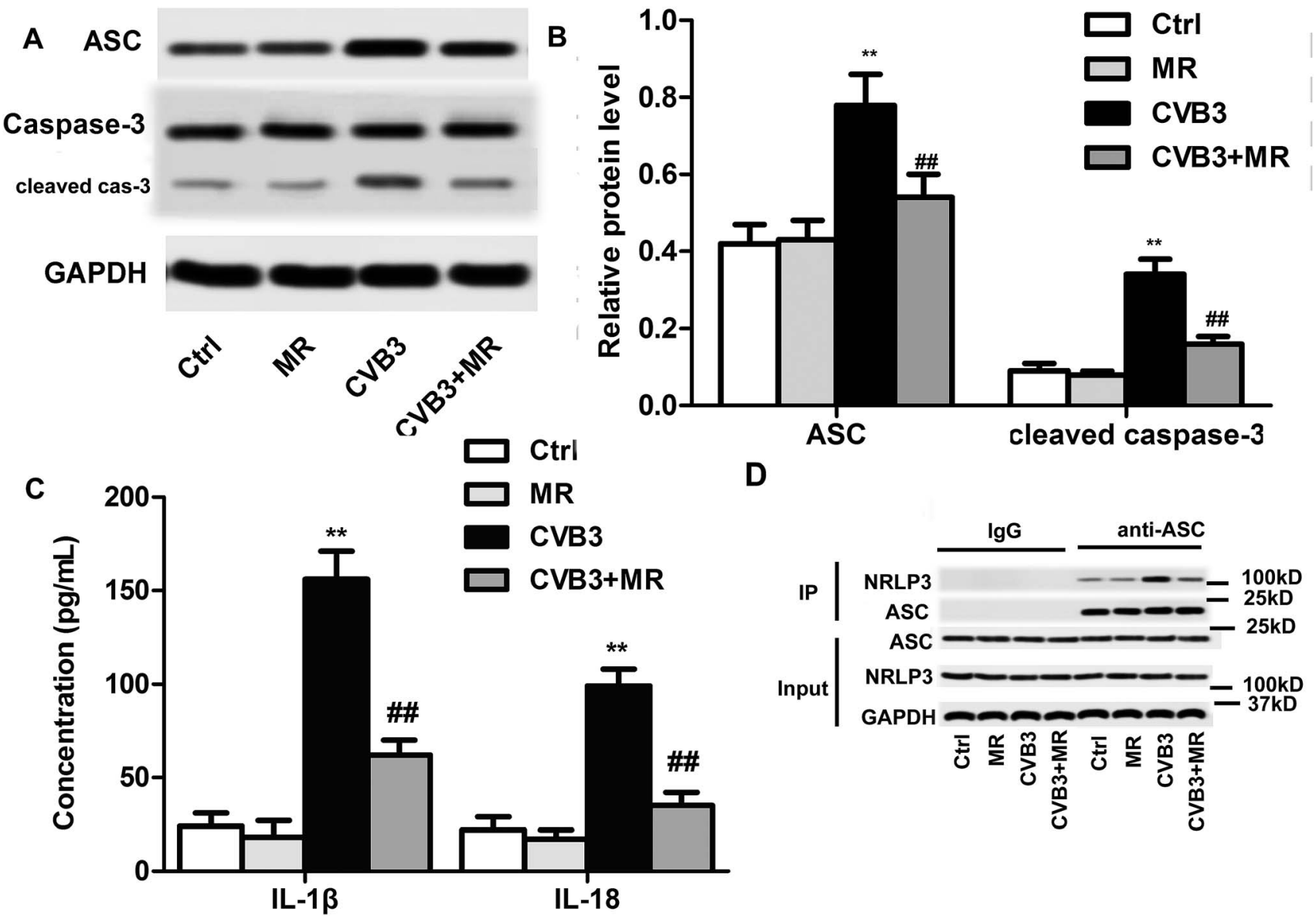

Fig. 6 Underlying mechanism of MR alleviating myocardial damage. (A) Expression of ASC and caspase-3 in heart tissues of different rats evaluated by western blot assay, as a qualitative analysis. (B) Histogram showing the quantitative results. (C) The levels of pro-inflammatory cytokines IL- $\beta$ and IL-18 in heart tissues of each group determined by ELISA experiments. (D) IP was performed to determine the interaction between NLRP3 and ASC. ${ }^{* * P} P 0.01$ compared with control group, ${ }^{\# \#} P<0.01$ compared with control group. CVB3 $=$ coxsackievirus B3, MR $=$ morroniside, ASC = apoptosis-associated speck-like protein containing CARD.

treatment with MR. To verify that hypothesis, western blot assay and ELISA were applied to determine the expression of those genes in the hearts of each group. As exhibited in Fig. 6A, the levels of ASC and caspase- 3 in heart tissues have been significantly elevated after activation of the NLRP3 inflammasome by CVB3 induction. However, expression of ASC and caspase-3 can be recovered to nearly normal physiological levels by treating the CVB3-induced rats with appropriate dosage of MR. For the IL-1 family of cytokines, IL- $\beta$ and IL-18, they showed a similar expression to ASC and caspase- 3 with the levels of IL- $\beta$ and IL-18 in hearts of CVB3-induced rats treated with MR being remarkably lower than those of the untreated ones. These results together suggest that myocardial damage was alleviated by MR through down-regulating the expression of ASC and caspase-3 which in turn leads to a significant decrease of the levels of pro-inflammatory cytokines IL- $\beta$ and IL-18.

\section{Conclusion}

In the present study, to alleviate the myocardial damage of CVB3-infected rats, MR was introduced followed by detailed investigation of the treatment efficacy and underlying mechanism. For performing experiments, various technologies and methods such as RT-PCR, western blot, ELISA, and H\&E or TUNEL staining have been applied. The obtained results indicated that the myocardial damage markers and cardiac function indexes of CVB3-induced rats could be recovered to normal levels after MR therapy. The results of the mechanistic study showed that the inhibition of NLRP3 inflammasome activation, the main pathogenesis of CVB3-induced viral myocarditis, by MR led to a significant down-regulation of downstream genes including ASC, caspase-3, IL- $\beta$, and IL-18. Moreover, the safety evaluation of MR in normal rats showed that the related indexes of heart tissues after treatment with MR displayed negligible difference from the control group. In summary, improving cardiac function via restraining NLRP3 inflammasome activation might represent the most promising strategy for alleviating CVB3-induced myocardial damage.

\section{Discussion}

Myocarditis, mostly induced by viral infections, represents one of the predominate causes of heart failure. ${ }^{33}$ As a serious clinical condition, viral myocarditis is always correlated with sudden 
cardiac death in children and young adults whose ages are below 40 years old. ${ }^{34}$ The fatality of viral myocarditis is mainly ascribed to its easily progressing to a dilated phenotype inflammatory dilated cardiomyopathy which is characterized by poor prognosis. ${ }^{35}$

CVB3, as has been demonstrated by many epidemiological studies, is the most common pathogen for viral myocarditis. ${ }^{33,36}$ Although the regulatory mechanisms underlying CVB3-induced dilated cardiomyopathy are still poorly defined, accumulating evidences suggest that the main pathologic factor of viral myocarditis is overwhelming inflammation. ${ }^{36}$ Importantly, it has been demonstrated that NLRP3 inflammasome activation induced by CVB3 infection is a central player in developing myocardial dysfunction. ${ }^{37}$ In this case, inhibition of NLRP3 inflammasome activation is suggested as the most promising strategy for alleviating CVB3-induced myocardial damage.

Herein, MR was selected as a model drug against viral myocarditis for its great potential in suppressing inflammation by depressing the activation of caspase and production of ROS. ${ }^{24}$ For verification, various experiments have been performed. MAP, HR, and LVSP are the main indexes to evaluate cardiac function. In the rats with myocardial damage, those indexes were significant lower than the normal levels. Consistent with previous reports, the CVB3-infected rats in the present study displayed lower levels of MAP, HR, and LVSP compared with the normal rats. However, such symptoms could be remarkably remitted by treating with $\mathrm{MR}$ as demonstrated in Fig. 2. Moreover, the results of H\&E staining and TUNEL experiments further confirmed that MR could obviously alleviate the myocardial damage induced by CVB3 infection.

Restraining NLRP3 inflammation activation has been identified as the most efficient strategy to alleviate CVB3-induced myocardial damage. To examine whether MR has an effect on the expression of NLRP3, RT-PCR experiments and western blot assay were conducted. The results in Fig. 5 illustrate that the levels of NLRP3 in CVB3-induced rats were notably higher than those in normal ones which resulted in serious heart tissue damage (Fig. 3A) and down-regulation of Bcl-2 expression (Fig. 3B). However, the hyperactive NLRP3 could be significantly suppressed by MR and finally led to an improvement of cardiac function by reducing the myocardial damage makers including $\mathrm{Mb}, \mathrm{CK}-\mathrm{MB}$, and cTn I.

The pro-inflammatory cytokines IL- $\beta$ and IL-18 are the downstream genes of NLRP3 and play an important role in exacerbating inflammatory responses and inducing cardiodepressive effects. ${ }^{\mathbf{1 3 , 1 4}}$ As demonstrated above, NLRP3 inflammasome activation can be depressed by MR therapy. In this case, the levels of downstream genes of NLRP3 in heart tissues of CVB3-infected rats would definitely be influenced by the MR treatment. As demonstrated in Fig. 6, the expression of ASC, caspase-3, IL- $\beta$ and IL-18 was obviously silenced after treatment with appropriate concentration of MR.

Importantly, to evaluate the safety of MR to normal heart tissues, rats uninfected with CVB3 were also subjected to treatment with the agents. Subsequently, many indexes including cardiac function indexes, myocardial damage makers, and various gene or protein expressions were determined by the same method as above. Results obtained from the present study showed that the histopathology and symptoms of normal rats treated with MR exhibited negligible changes compared with the non-treated control group, indicating a satisfactory biocompatibility of MR.

\section{Conflicts of interest}

No.

\section{Abbreviations}

$\begin{array}{ll}\text { CVB3 } & \text { Coxsackievirus B3 } \\ \text { NLRP3 } & \text { NOD-like receptor pyrin domain-containing } 3 \\ \text { MR } & \text { Morroniside } \\ \text { MAP } & \text { Mean arterial pressure } \\ \text { HR } & \text { Heart rate } \\ \text { LVSP } & \text { Left ventricular systolic pressure } \\ \text { Mb } & \text { Muscle hemoglobin } \\ \text { CK-MB } & \text { Creatine kinase isoenzyme } \\ \text { cTn I } & \text { Cardiac troponin I } \\ \text { H\&E } & \text { Hematoxylin and eosin }\end{array}$

\section{References}

1 F. Dominguez, U. Kuhl, B. Pieske, P. Garcia-Pavia and C. Tschope, Rev. Esp. Cardiol., 2016, 69, 178-187.

2 A. L. Caforio, S. Pankuweit, E. Arbustini, C. Basso, J. GimenoBlanes, S. B. Felix, M. Fu, T. Helio, S. Heymans, R. Jahns, K. Klingel, A. Linhart, B. Maisch, W. McKenna, J. Mogensen, Y. M. Pinto, A. Ristic, H. P. Schultheiss, H. Seggewiss, L. Tavazzi, G. Thiene, A. Yilmaz, P. Charron and P. M. Elliott, Eur. Heart J., 2013, 34, 2636-2648.

3 D. Fairweather and N. R. Rose, Methods, 2007, 41, 118-122.

4 C. Badorff, B. Fichtlscherer, R. E. Rhoads, A. M. Zeiher, A. Muelsch, S. Dimmeler and K. U. Knowlton, Circulation, 2000, 102, 2276-2281.

5 H. Zhang, Y. Yue, T. Sun, X. Wu and S. Xiong, Sci. Rep., 2017, 7, 42162.

6 M. Kawaguchi, M. Takahashi, T. Hata, Y. Kashima, F. Usui, H. Morimoto, A. Izawa, Y. Takahashi, J. Masumoto, J. Koyama, M. Hongo, T. Noda, J. Nakayama, J. Sagara, S. Taniguchi and U. Ikeda, Circulation, 2011, 123, 594-604.

7 J. W. Mason, Cardiovasc. Res., 2003, 60, 5-10.

8 M. P. Reyes and A. M. Lerner, Prog. Cardiovasc. Dis., 1985, 27, 373-394.

9 X. Li, F. Li, Y. Chu, X. Wang, H. Zhang, Y. Hu, Y. Zhang, Z. Wang, X. Wei, W. Jian, X. Zhang and F. Yi, Cell. Physiol. Biochem., 2013, 32, 1857-1866.

10 A. Sabbah, T. H. Chang, R. Harnack, V. Frohlich, K. Tominaga, P. H. Dube, Y. Xiang and S. Bose, Nat. Immunol., 2009, 10, 1073-1080.

11 F. S. Sutterwala, S. Haasken and S. L. Cassel, Ann. N. Y. Acad. Sci., 2014, 1319, 82-95.

12 A. Abbate, F. N. Salloum, E. Vecile, A. Das, N. N. Hoke, S. Straino, G. G. Biondi-Zoccai, J. E. Houser, I. Z. Qureshi, E. D. Ownby, E. Gustini, L. M. Biasucci, A. Severino, 
M. C. Capogrossi, G. W. Vetrovec, F. Crea, A. Baldi, R. C. Kukreja and A. Dobrina, Circulation, 2008, 117, 26702683.

13 B. W. Van Tassell, S. Toldo, E. Mezzaroma and A. Abbate, Circulation, 2013, 128, 1910-1923.

14 C. F. McTiernan, B. H. Lemster, C. Frye, S. Brooks, A. Combes and A. M. Feldman, Circ. Res., 1997, 81, 493-503.

15 B. J. Pomerantz, L. L. Reznikov, A. H. Harken and C. A. Dinarello, Proc. Natl. Acad. Sci. U. S. A., 2001, 98, 2871-2876.

16 Y. Liu, K. Lian, L. Zhang, R. Wang, F. Yi, C. Gao, C. Xin, D. Zhu, Y. Li, W. Yan, L. Xiong, E. Gao, H. Wang and L. Tao, Basic Res. Cardiol., 2014, 109, 415.

17 B. Yu, G. Zhang, Y. An and W. Wang, Korean J. Physiol. Pharmacol., 2018, 22, 17-21.

18 B. Yu and W. Wang, Inflammation, 2018, 41, 432-436.

19 C. Y. Li, L. Li, Y. H. Li, H. X. Ai and L. Zhang, China J. Chin. Mater. Med., 2005, 30, 1667-1670.

20 X. Li, C. Huo, Q. Wang, X. Zhang, X. Sheng and L. Zhang, J. Pharm. Biomed. Anal., 2007, 45, 268-274.

21 W. Wang, W. Huang, L. Li, H. Ai, F. Sun, C. Liu and Y. An, Cell. Mol. Neurobiol., 2008, 28, 293-305.

22 H. Wang, P. Gao, L. Jing, X. Qin and X. Sun, Biochimie, 2012, 94, 1951-1959.

23 W. Wang, F. Sun, Y. An, H. Ai, L. Zhang, W. Huang and L. Li, Eur. J. Pharmacol., 2009, 613, 19-23.

24 W. Wang, J. Xu, L. Li, P. Wang, X. Ji, H. Ai, L. Zhang and L. Li, Brain Res. Bull., 2010, 83, 196-201.

25 M. Li, W. Wang, P. Wang, K. Yang, H. Sun and X. Wang, Molecules, 2010, 15, 7403-7414.

26 I. Almendros, R. Farre, M. Torres, M. R. Bonsignore, M. Dalmases, J. Ramirez, D. Navajas and J. M. Montserrat, Sleep Medicine, 2011, 12, 1037-1040.
27 A. M. Al-Taweel, M. Raish, S. Perveen, G. A. Fawzy, A. Ahmad, M. A. Ansari, S. Mudassar and M. A. Ganaie, Phytomedicine, 2017, 34, 67-75.

28 M. M. Abdel-Daim, O. E. Kilany, H. A. Khalifa and A. A. M. Ahmed, Cancer Chemother. Pharmacol., 2017, 80, 745-753.

29 X. Ye, T. Shen, J. Hu, L. Zhang, Y. Zhang, L. Bao, C. Cui, G. Jin, K. Zan, Z. Zhang, X. Yang, H. Shi, J. Zu, M. Yu, C. Song, Y. Wang, S. Qi and G. Cui, Exp. Neurol., 2017, 292, $46-55$.

30 Y. Wang, B. Gao and S. Xiong, Am. J. Physiol.: Heart Circ. Physiol., 2014, 307, H1438-H1447.

31 K. Fuse, G. Chan, Y. Liu, P. Gudgeon, M. Husain, M. Chen, W. C. Yeh, S. Akira and P. P. Liu, Circulation, 2005, 112, 2276-2285.

32 G. Schoch, H. Seeger, J. Bogousslavsky, M. Tolnay, R. C. Janzer, A. Aguzzi and M. Glatzel, PLoS Med., 2006, 3, e14.

33 G. Li-Sha, L. Li, Z. De-Pu, S. Zhe-Wei, G. Xiaohong, C. GuangYi, L. Jia, L. Jia-Feng, C. Maoping and L. Yue-Chun, Front. Pharmacol., 2018, 9, 182.

34 W. Gou, Z. Zhang, C. Yang and Y. Li, Exp. Cell Res., 2018, 366, 41-48.

35 K. Miteva, K. Pappritz, M. Sosnowski, M. El-Shafeey, I. Muller, F. Dong, K. Savvatis, J. Ringe, C. Tschope and S. Van Linthout, Sci. Rep., 2018, 8, 2820.

36 Y. Wang, L. Jia, J. Shen, Y. Wang, Z. Fu, S. A. Su, Z. Cai, J. A. Wang and M. Xiang, PLoS Pathog., 2018, 14, e1006872.

37 C. Tschope, I. Muller, Y. Xia, K. Savvatis, K. Pappritz, S. Pinkert, D. Lassner, M. M. Heimesaat, F. Spillmann, K. Miteva, S. Bereswill, H. P. Schultheiss, H. Fechner, B. Pieske, U. Kuhl and S. Van Linthout, Circ.: Heart Failure, 2017, 10(9), e003870. 